\title{
Article \\ Distributed Deep Fusion Predictor for a Multi-Sensor System Based on Causality Entropy
}

\author{
Xue-Bo Jin ${ }^{1,2, * \mathbb{D}}$, Xing-Hong Yu ${ }^{1,2}$, Ting-Li Su ${ }^{1,2, *}$, Dan-Ni Yang ${ }^{3}$, Yu-Ting Bai ${ }^{1,2} \mathbb{D}$, Jian-Lei Kong ${ }^{1,2}$ and \\ Li Wang 1,2,*
}

check for

updates

Citation: Jin, X.-B.; Yu, X.-H.;

Su, T.-L.; Yang, D.-N.; Bai, Y.-T.; Kong, J.-L.; Wang, L. Distributed Deep

Fusion Predictor for a

Multi-Sensor System Based on Causality

Entropy . Entropy 2021, 23, 219.

https://doi.org/10.3390/e23020219
1 Artificial Intelligence College, Beijing Technology and Business University, Beijing 10048, China; yuxinghong@st.btbu.edu.cn (X.-H.Y.); baiyuting@btbu.edu.cn (Y.-T.B.); kongiianlei@btbu.edu.cn (J.-L.K.)

2 China Light Industry Key Laboratory of Industrial Internet and Big Data Beijing Technology and Business University, Beijing 10048, China

3 Electrical and Information Engineering College, Tianjin University, Tianjin 300072, China; 18693803229@163.com

* Correspondence: jinxuebo@btbu.edu.cn (X.-B.J.); sutingli@btbu.edu.cn (T.-L.S.); wangli@th.btbu.edu.cn (W.L.)
Academic Editor: Quan Min Zhu, Giuseppe Fusco, Jing Na, Weicun Zhang and Ahmad Taher Azar

Received: 11 January 2021

Accepted: 7 February 2021

Published: 11 February 2021

Publisher's Note: MDPI stays neutral with regard to jurisdictional claims in published maps and institutional affiliations.

Copyright: (c) 2021 by the authors. Licensee MDPI, Basel, Switzerland. This article is an open access article distributed under the terms and conditions of the Creative Commons Attribution (CC BY) license (https:// creativecommons.org/licenses/by/ $4.0 /)$.
Abstract: Trend prediction based on sensor data in a multi-sensor system is an important topic. As the number of sensors increases, we can measure and store more and more data. However, the increase in data has not effectively improved prediction performance. This paper focuses on this problem and presents a distributed predictor that can overcome unrelated data and sensor noise: First, we define the causality entropy to calculate the measurement's causality. Then, the series causality coefficient (SCC) is proposed to select the high causal measurement as the input data. To overcome the traditional deep learning network's over-fitting to the sensor noise, the Bayesian method is used to obtain the weight distribution characteristics of the sub-predictor network. A multi-layer perceptron (MLP) is constructed as the fusion layer to fuse the results from different subpredictors. The experiments were implemented to verify the effectiveness of the proposed method by meteorological data from Beijing. The results show that the proposed predictor can effectively model the multi-sensor system's big measurement data to improve prediction performance.

Keywords: series causality analysis; Bayesian LSTM; multi-sensor system; meteorological data; big measurement data; deep fusion predictor

\section{Introduction}

Measurements have been obtained and saved in many multi-sensor systems, such as mobile robots [1], unmanned aerial vehicles (UAVs) [2,3], smart agriculture [4,5], air quality monitoring systems [6,7], etc. It is very meaningful to analyze these data and understand and predict the information in the sensor system [8], for example the analysis and prediction of meteorological elements in precision agriculture or environmental management systems [9]. Furthermore, in terms of environmental governance, the prediction for air pollution sources such as PM2.5 has played an important role [10-13].

Recently, more measurements have been collected with the development of sensor technology. Therefore, in a multi-sensor system, big data analysis has become a new research area. These data have two characteristics: noisy and numerous [14]. For example, the collected and saved meteorological data are big data and include many variables, such as temperature, wind, rainfall, humidity, etc. Further, they are related to each other [15]. However, the correlation between each type of variable is different: some of them have a strong correlation, but some have a low correlation.

In general, more data can provide more information. For big data, deep learning can extract hidden information to make more accurate predictions [16]. The recent research has proven that the recurrent neural network (RNN) and its improved version are widely used 
in regression prediction problems with better nonlinear modeling ability compared with the classical regression method.

We can find that the network has become larger and more complex due to the massive amount of data. However, because of the amount of data, the network's training time is getting longer. To make matters worse, the increase of the input data does not improve the prediction performance; on the contrary, it decreases.

This paper focuses on how to use this big noisy data in the multi-sensor system to efficiently improve prediction performance. This paper mainly aims at multi-sensor systems, proposes a causal entropy method for feature selection, and constructs a distributed forward multi-step prediction framework based on Bayesian deep learning theory. In this way, the dimensionality reduction of high-dimensional data feature selection is realized, and the problem of data noise affecting deep network training is initially overcome. The rest is organized as follows: Section 2 summarizes current prediction models and describes the main contribution of this paper. Section 3 proposes a distributed deep learning network predictor, and Section 4 describes the experiments and results to verify the performance of our predictor. We draw conclusions in Section 5.

\section{Related Works}

\subsection{The Methods for Prediction}

Prediction is to analyze historical data and obtain the trend of the future. With the development of computer storage technology and sensor technology, the prediction of measurement data in a multi-sensor system has been widely used in many fields. It has become a hot topic of research. The traditional prediction methods require prior knowledge of the data, such as exponential smoothing [17], moving average (MA) [18], auto-regression (AR) [19], auto-regressive integrated moving average (ARIMA) [20], etc. In practical systems, the traditional prediction methods cannot obtain a high accuracy prediction result due to the system's complexity.

For nonlinear input data, shallow machine learning methods obtain model parameters through training, such as support vector machines (SVMs) [21], the echo state network (ESO) [22], Boltzmann machines (BMs) [23], shallow artificial neural networks (ANNs) [24], generalized regression neural networks (GRNNs) [25], etc., which avoids the requirement of prior knowledge of the data. However, because of their simple structure, they cannot process large amounts of data.

With the development of the depth of the neural network, the hidden information in the massive data can be extracted to make more accurate predictions. The recurrent neural network (RNN) [26] and its improved versions, such as long short-term memory (LSTM) [27], etc., is widely used for regression prediction problems, demonstrating its superior nonlinear modeling capabilities. For example, a gated recurrent unit (GRU) network [28] and Bi-LSTM [29] were proposed to improve LSTM. Furthermore, researchers [30,31] have combined the one-dimensional convolutional neural network (CNN) with LSTM to predict the time series data. System identification is the theory, and the methods of establishing the mathematical models of dynamical systems [32-36] and some identification approaches can be used to establish the prediction models and soft sensor models [37-42] for various application problems.

\subsection{The Method to Calculate Causality and Correlation}

Undoubtedly, deep neural networks are currently the best solution to the big data prediction problem of multi-sensor systems. However, we found that the network's ability to predict does not increase as the amount of input variable increases. On the contrary, sometimes, the larger the amount of input measurement data from multi-sensor system, the worse performance the prediction obtains. This is contrary to what we have always believed: one advantage of the deep learning network is that it has comprehensive and robust learning capabilities for big data. 
We believe one of the reasons is that the data contain too much low-relevance information; the increase in the amount of data leads to a decrease in the ratio of useful information. The weights of training for the neural network and the diluted information make the network's convergence more difficult, so the prediction performance cannot be developed, but is even reduced. Therefore, we think the data with a high correlation and strong causality with the target variable should be selected as the network's input data, rather than just increasing the number.

Then, we describe the correlation degree method and discuss a causal correlation method for measuring variables suitable for multi-sensor systems to measure big data. The Pearson correlation coefficient (PCC) [43] and Spearman correlation coefficient $[44,45]$ have been used for such a problem. The former can be used to find the linear relationship between the two variables. For the data, the features are continuous and conform to a positive distribution; the linear relationship between the two variables can be mined by the PCC [46]. Jing et al. [47] selected the characteristic sub-sequence by PCC to improve the prediction accuracy when forecasting photo-voltaic power output. Lin et al. [48] built a hybrid model framework using the stacking scheme of integrated learning by PCC between different models. As for the prediction problem, PCC requires a known prediction target variable, so it cannot be applied for predicting.

Spearman's correlation coefficient is mainly used to solve problems related to sequential data. It applies to two variables with an order relationship. Another kind of correlation analysis method is called the Kendall correlation coefficient [49], which is suitable for sequenced variables or evenly spaced data that do not satisfy the assumption of a normal distribution. This method is usually calculated for a piece of sequence data, and it cannot obtain an effective correlation between the input and output for large amounts of data.

Contreras-Reyes et al. [50] used the frequency-domain Granger-causality method to test the statistical significance of causality between two time series and determine the direction of causality on the drivers of pelagic species' biological indicators. Since the Granger causality coefficient is used in two stationary time series, its application is limited. Podobnik et al. [51] proposed a detrending cross-correlation analysis method to explore the correlation between two non-stationary series. It shows that effectively measuring the correlation between two variables can help analyze the change characteristics of one of the variables.

The current methods to calculate the correlation and causality rely on the predicted result and cannot be applied to the prediction problem of multi-sensor systems.

\subsection{The Bayesian Deep Learning Network}

The big data measured by sensors contain noise, which is another reason for the degradation of prediction performance based on deep learning networks. Traditional neural network training obtains the fixed weights and biases, which are easily disturbed by noise $[52,53]$. On the one hand, the noise makes it difficult to converge the network, that is the loss of the network is larger. On the contrary, if the noise is also learned as a certain value until a small loss value is obtained, it will cause the problem of overfitting [54].

Suppose we use the data distribution to train the network and obtain weights and deviations to express the input data's distribution characteristics. In that case, the problem of overfitting will be avoided. Based on the distribution characteristics of the weights and deviations, the obtained neural network is a group. The output is also a group of prediction outputs with distribution characteristics, improving the prediction results' reliability. Based on this research question, the Bayesian deep learning network came into being [55]. Through Monte Carlo sampling, the Bayesian deep learning network trains the network several times and takes the average of all losses, then uses it for backpropagation to obtain the distribution of weights and deviations [56].

The Bayesian method has been used in many application systems, such as indoor tracking [57], robot systems [58], etc. The Bayesian deep learning network has been applied in modeling with noisy data, and some results have been obtained. For example, 
Li et al. [59] integrated uncertainties by defining the Bayesian deep learning framework, in which a sequential Bayesian boosting algorithm is used to improve the estimation accuracy. Another example is [60], where a Bayesian framework was proposed to model the valence predictions.

\subsection{Innovation}

Aiming at the problem of improving the prediction performance based on the huge amount measurement data in a multi-sensor system, this paper provides a distributed deep prediction network. To solve the contradiction between data volume and performance and the influence of data noise on prediction performance, the innovation of this paper lies in the following:

(1) A series causality entropy method is developed to select the related input data for the neural network. Compared with the PCC [48], Spearman correlation [45], and the Kendall correlation coefficient method [61], the method does not depend on the prediction results and is suitable for prediction problems based on measurement data in multi-sensor systems.

(2) A distributed prediction framework is proposed, in which Bayesian training is used to suppress the noise impact of the data, and the prediction based on the selected input data is fused by a nonlinear fusion network. Compared with the classical LSTM [27], GRU [28], CNN-LSTM [11], the conv-LSTM [30] predictor, etc., the proposed method outperforms them in its prediction performance.

\section{Distributed Deep Fusion Predictor}

\subsection{Series Causality Entropy}

In a multi-sensor system, we can set up multiple sensors to obtain a variety of measurement data. For example, in the system given in Figure 1, we use four sensors to obtain four types of measurement data, and they will be used as candidate input data for the deep network. The prediction task is for Measurement 1 , and we will predict its future trend.

Firstly, we will consider the method to select the input data for the networks. Obviously, the principle of selecting data is to select those measurement data that are most causal for the future trend of Measurement 1 . As for the prediction problem, it can be defined as the series causality between the historical data and the future data.

We give the following definition about the causality entropy to calculate the measurement's causality between two data named $X$ and $Y$ :

$$
C E(X, Y)=\frac{1}{N-1} \sum_{i=1}^{N}\left(\frac{X(i)-\bar{X}}{\sigma_{X}}\right) \times \log \left(\frac{Y(i)-\bar{Y}}{\sigma_{Y}}\right)
$$

where $\bar{X}$ and $\bar{Y}$ are the mean of $X(i)$ and $Y(i), i=1,2, \ldots \ldots, N$, respectively, and $\sigma_{X}$ and $\sigma_{Y}$ are the standard deviation of $X(i)$ and $Y(i)$. We can see that $C E(X, Y)$ can be positive or negative. When it is a positive number, it indicates that the two data are positively supporting. Otherwise, it is negatively supporting.

This calculating correlation method cannot be directly applied to obtain the correlation of prediction problems. Because the measured data and their prediction are considered in the prediction problem, therefore, we cannot calculate $C E$ when the prediction is not yet available. Secondly, since the step length of the measurement data $I$ is different from the predicted step length $J$, the number of data points $N$ in Equation (1) cannot be used.

Therefore, we propose the following series causality coefficient (SCC) for the measurement in the multi-sensor system. Suppose the measured data are represented by $X_{m}(i)$, where $m=1,2, \ldots \ldots, M$ is the sensor number to obtain the measurement and $i=1,2, \ldots \ldots, I$ is the step number of historical data used for prediction. The target data to be predicted are represented by $Y(j)$, where $j=1,2, \ldots \ldots, J$ is the step number of prediction. We revised the method to calculate coefficient Equation (1) as the following. 


$$
S_{m}=\frac{1}{K-1} \sum_{i=1, j=1}^{K}\left|\frac{X_{m}(i)-\overline{X_{m}}}{\sigma_{X_{m}}}\right| \times \log \left|\frac{Y(j)-\bar{Y}}{\sigma_{Y}}\right|
$$

where $m=1,2, \ldots . M$ is the sensor number to obtain the measurement, $K=\min (I, J)$, $\overline{X_{n}}$ and $\bar{Y}$ are the mean of $X m(i)$ and $Y(i), i=1,2, \ldots \ldots K$, respectively, and $\sigma_{X_{m}}$ and $\sigma_{Y}$ are the standard deviation of $\overline{X_{n}}$ and $\bar{Y}$. We can find that Equation (2) still has the prediction $Y(i)$, which is unknown data. To eliminate $Y(i)$ in Equation (2), we modify Equation (2) by normalization. The normalized SCC of each measurement can be obtained by the following.

$$
S C C_{m}^{*}=\frac{S_{m}}{S_{1}+S_{2}+\ldots+S_{M}}=\frac{\sum_{i=1}^{K}\left|\frac{X_{m}(i)-\overline{X_{m}}}{\sigma_{X_{m}}}\right|}{\sum_{m=1}^{M} \sum_{i=1}^{K}\left|\frac{X_{m}(i)-\overline{X_{m}}}{\sigma_{X_{m}}}\right|}
$$

From Equation (3), we can conclude that the value of SCC is between zero and one; the larger the $S C C$, the higher the causality is. For example, when the value is zero, it means that the feature is not useful for predicting the target variable. We can see that the SCC given by Equation (3) omits the calculation process for the prediction $Y(i)$.

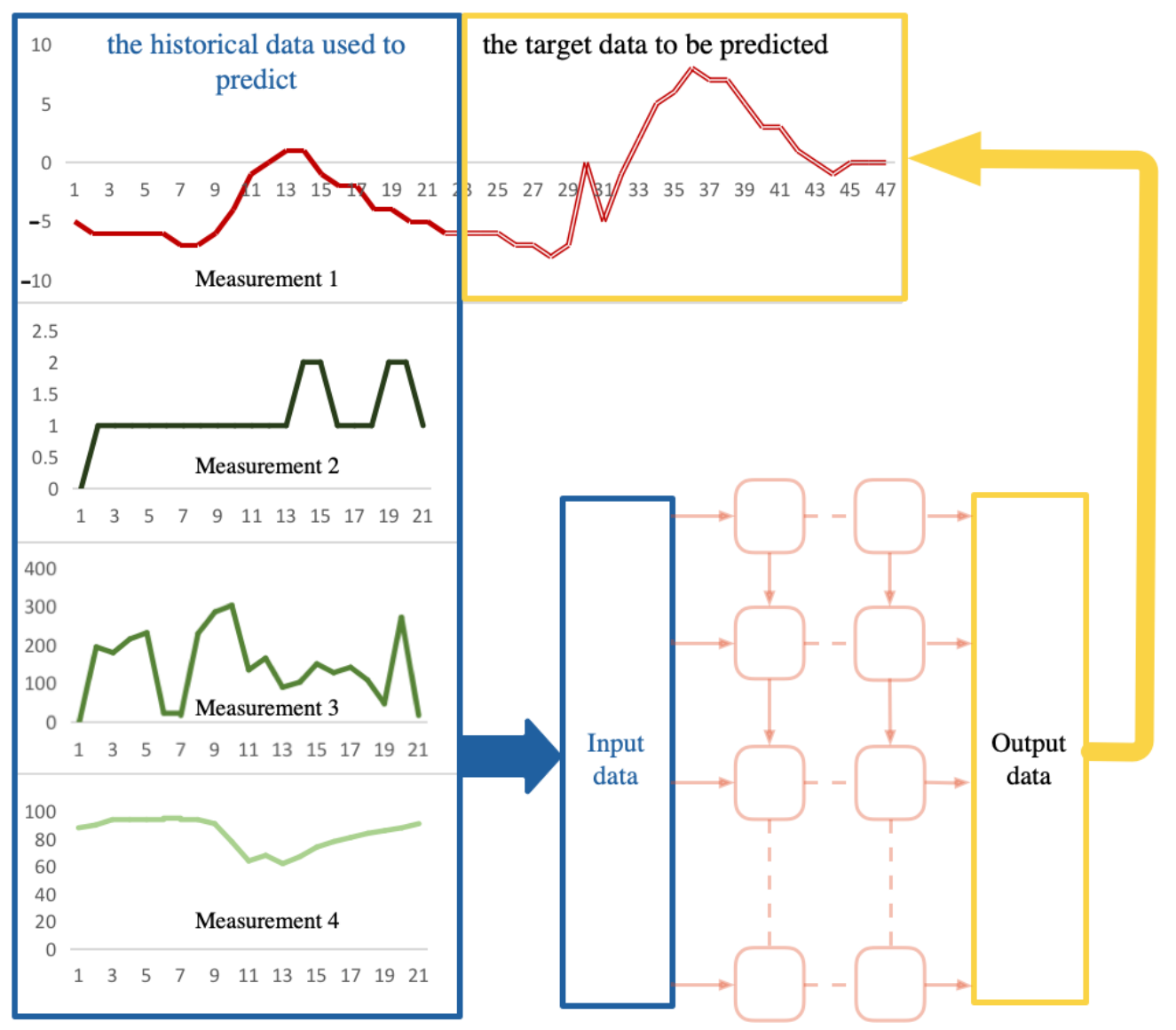

Figure 1. Relationship between the target variables to be predicted and the input variables.

We give the following examples to illustrate the SCC obtained by Equation (3). Meteorological data are used, including temperature, wind direction, wind force, rainfall, and humidity, which are used to predict the future temperature. We have five measurements, so according to Equation (3), $M$ is five. We set $K=24$, then $S C C_{m}$ can be obtained. $\mathrm{X}$ is set to five meteorological elements separately, and $\mathrm{Y}$ is the future temperature to be predicted. The result is shown in Table 1 . To clearly illustrate the difference of $S C C_{m}$, we visualize them as Figure 2. 
Table 1. The order of the SCC between variables to be predicted.

\begin{tabular}{cc}
\hline Measurement & Series Causality Coefficient (SCC) \\
\hline Temperature & 0.3673 \\
Humidity & 0.3259 \\
Wind force & 0.175 \\
Wind direction & 0.1318 \\
Rainfall & 0 \\
\hline
\end{tabular}

It can be seen from Table 1 and Figure 2 that the causality between historical temperature data and their future prediction is the largest, which is 0.3673 . Next is humidity. We get the SSC as 0.3259 between historical humidity and future temperature. Compared with them, the causal relationship between wind force and wind direction with the future temperature is smaller. The data can also reflect no causal relationship between rainfall and temperature, for which we obtain a zero SSC.

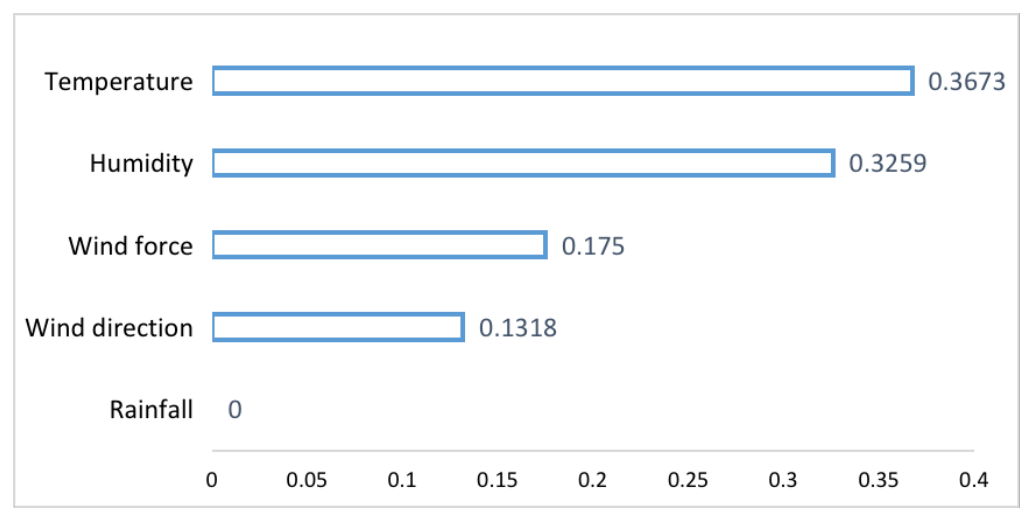

Figure 2. SCC between different measurements and predictions.

Further, we can get the following conclusions. If all the data are used for training, rainfall data can only cause the network to reduce the training's convergence and the temperature prediction performance. Therefore, rainfall data must be eliminated and cannot be used as the input for network training and prediction. Regarding wind force and wind direction data, because of their low causality, even as the network's input data, the performance improvement of the prediction results is limited. It will increase the training time of the network. On the contrary, the humidity data have a high causal correlation with the future temperature. Therefore, using the historical temperature data and humidity data to predict the future temperature may achieve better performance than just using temperature data. The experiments in Section 4 will verify the above points.

\subsection{Bayesian LSTM as the Sub-Predictor}

The LSTM cell is used in this paper, which is composed of three gating units, i.e., input gate, forget gate, and output gate. The calculation process is the following:

$$
\begin{aligned}
f_{t} & =\sigma\left(W_{f x} x_{t}+W_{f h} h_{t-1}+b_{f}\right) \\
i_{t} & =\sigma\left(W_{i x} x_{t}+W_{i h} h_{t-1}+b_{i}\right) \\
\bar{c}_{t} & =\tanh \left(W_{c x} x_{t}+W_{c h} h_{t-1}+b_{c}\right) \\
c_{t} & =f_{t} \cdot c_{t-1}+i_{t} \cdot \bar{c}_{t} \\
o_{t} & =\sigma\left(W_{o x} x_{t}+W_{o h} h_{t-1}+b_{o}\right) \\
h_{t} & =o_{t} \cdot \tanh \left(c_{t}\right)
\end{aligned}
$$

where $t$ is the current moment to predict, $w=\left[W_{f x}, W_{f h}, W_{i x}, W_{c x}, W_{o x}, W_{o h}\right]$ are the weights, and $b=\left[b_{f}, b_{i}, b_{o}\right]$ are the biases. $c_{t}$ is the hidden state, and $h_{t}$ is the output of 
the LSTM cell. The cells can be placed as several layers with different input and output cells depending on the number of input and output steps of the prediction. The structure of the network is shown in Figure 3. The input data $x$ are the given data used to predict the future trend, where $x=[X(1), X(2), \ldots \ldots, X(I)]$ are the input data at each moment with the number of data $I$, and $x_{t}=\left[X_{t}(1), X_{t}(2), \ldots \ldots, X_{t}(I)\right]$ are the input data at the current moment $t$. The output of the last layer can be set as the output of the LSTM network, named as $y$. For the training process, we have $y=[Y(1), Y(2), \ldots \ldots, Y(J)]$, and at the current moment $t$, we have $y_{t}=\left[Y_{t}(1), Y_{t}(2), \ldots \ldots, Y_{t}(J)\right]$.

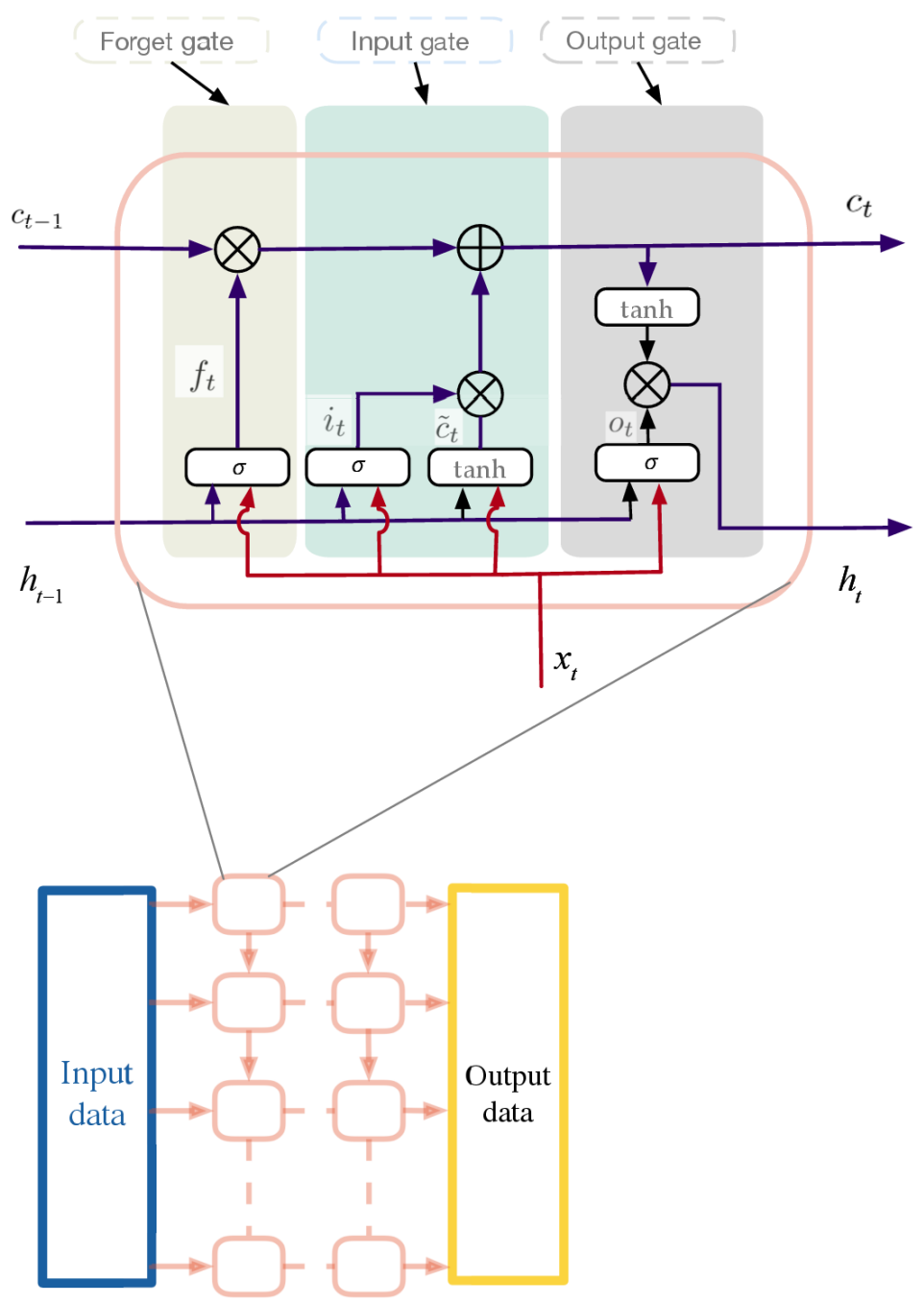

Figure 3. LSTM cell and its networks.

In the normal LSTM network, the parameters, including all the weights and biases, are constants. The Bayesian LSTM can get the weight and bias as a random distribution, not a certain value. Each parameter obtained by the Bayesian LSTM network training is the mean and variance according to the distribution of the weights and biases. The difference between the normal LSTM network and the Bayesian LSTM network is shown in Figure 4.

The LSTM neural network can be seen as a probabilistic model $P(y \mid x, \theta)$ : a probability given an input $x \in \mathbb{R}^{p}$ to each possible output $y \in Y$, using the set of parameters $\theta$ including weights $\mathrm{w}$ and biases $b$, i.e., $\theta=[w, b]$. We denote the training data $x$ and $y$ as $D$, i.e., $D=[x, y]$. 


\begin{tabular}{|c|c|c|c|}
\hline$W_{f h}$ & $W_{i x}$ & $W_{c x}$ & $W_{o x}$ \\
\hline$W_{f h}$ & $W_{i h}$ & $W_{c h}$ & $W_{o h}$ \\
\hline$b_{f}$ & $b_{i}$ & $b_{c}$ & $b_{o}$ \\
\hline
\end{tabular}

(a)

\begin{tabular}{|c|c|c|c|}
\hline 0.4 & 0.8 & 0.02 & 0.3 \\
\hline 0.7 & 0.4 & 0.5 & 0.9 \\
\hline 0.01 & 0.1 & 0.6 & 0.1 \\
\hline
\end{tabular}

(b)

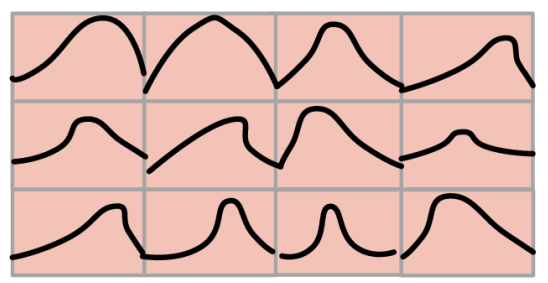

(c)
These are the weights and biases in each LSTM cell.

The normal LSTM networks obtain the weights and biases with a certain value after training.

Figure 4. The difference between the normal LSTM network and the Bayesian LSTM network. (a) The parameters in the LSTM; (b) the example of the parameters in the normal LSTM; (c) the example of the parameters in the Bayesian LSTM.

Given the training data $D$, Bayesian inference can be used to calculate the posterior distribution of weights $P(w \mid D)$ [62]. This distribution answers the predicted distribution of unknown data through the input data value: the predicted distribution of the input data $x$ is given by $P(y \mid x)=E_{P(\theta \mid D)}[P(y \mid x, \theta)]$. Until now, it is still difficult to find $P(w \mid D)$. The variational approximation to the Bayesian posterior distribution on the weights is a feasible method. Variational learning finds the parameters $(\mu, \sigma)$ of a distribution on the weights $q(\theta \mid \mu, \sigma)$ that minimizes the Kullback-Leibler (KL) divergence [63] with the true Bayesian posterior on the weights:

$$
(\mu, \sigma)^{*}=\arg \min _{\mu, \sigma} K L[q(\theta \mid \mu, \sigma) \| P(\theta \mid D)]
$$

According to the Bayesian theory,

$$
P(\theta \mid D)=\frac{P(D \mid \theta) P(\theta)}{P(D)}
$$

and the definition of the Kullback-Leibler (KL) divergence, Equation (5) can be transformed to:

$$
(\mu, \sigma)^{*}=\arg \min _{\mu, \sigma} \int q(\theta \mid \mu, \sigma) \log \frac{q(\theta \mid \mu, \sigma)}{P(\theta) P(D \mid \theta)} d \theta
$$

Note that we discarded $P(D)$ because it does not affect the optimized parameter solution. Then, the cost function is set as:

$$
\text { Loss }=\int q(\theta \mid \mu, \sigma) \log \frac{q(\theta \mid \mu, \sigma)}{P(\theta) P(D \mid \theta)} d \theta
$$


To keep the variance non-negative, we set it as $\sigma=\log (1+\exp (\rho))$. Set $\varepsilon$ as zero mean Gaussian white noise, i.e., $\varepsilon \sim \mathcal{N}(0,1)$. Then, we have $\theta=\mu+\log (1+\exp (\rho)) \otimes$ $\varepsilon$, where $\otimes$ is point-wise multiplication. Further, we can note that $q(\theta \mid \mu, \rho) d \theta=q(\varepsilon) d \varepsilon$, then the derivative of Equation (8) can be calculated as the following:

$$
\begin{aligned}
\frac{\partial}{\partial \mu} \text { Loss } & =\frac{\partial}{\partial \mu} \int q(\theta \mid \mu, \rho) \log \frac{q(\theta \mid \mu, \rho)}{P(\theta) P(D \mid \theta)} d \theta \\
\frac{\partial}{\partial \rho} \text { Loss } & =\frac{\partial}{\partial \rho} \int q(\theta \mid \mu, \rho) \log \frac{q(\theta \mid \mu, \rho)}{P(\theta) P(D \mid \theta)} d \theta
\end{aligned}
$$

Then, as for Equation (9), we have:

$$
\begin{aligned}
\frac{\partial}{\partial \mu} \text { Loss } & =\frac{\partial}{\partial \mu} \int q(\theta \mid \mu, \rho) \log \frac{q(\theta \mid \mu, \rho)}{P(\theta) P(D \mid \theta)} d \theta \\
& =\frac{\partial}{\partial \mu} \int \log \frac{q(\theta \mid \mu, \rho)}{P(\theta) P(D \mid \theta)} q(\theta \mid \mu, \rho) d \theta \\
& =\frac{\partial}{\partial \mu} \int \log \frac{q(\theta \mid \mu, \rho)}{P(\theta) P(D \mid \theta)} q(\varepsilon) d \varepsilon \\
& =\frac{\partial}{\partial \mu} \log \frac{q(\theta \mid \mu, \rho)}{P(\theta) P(D \mid \theta)} \int q(\varepsilon) d \varepsilon \\
& =\frac{\partial}{\partial \mu} \log \frac{q(\theta \mid \mu, \rho)}{P(\theta) P(D \mid \theta)}
\end{aligned}
$$

Similarly, Equation (10) can be derived further as the following:

$$
\frac{\partial}{\partial \rho} \text { Loss }=\frac{\partial}{\partial \rho} \log \frac{q(\theta \mid \mu, \rho)}{P(\theta) P(D \mid \theta)}
$$

Denote that:

$$
\text { Loss }=\log \frac{q(\theta \mid \mu, \rho)}{P(\theta) P(D \mid \theta)}=\log q(\theta \mid \mu, \rho)-\log P(\theta)-\log P(D \mid \theta)
$$

then we have:

$$
\begin{gathered}
\frac{\partial}{\partial \mu} \operatorname{Loss}=\frac{\partial \operatorname{Loss}}{\partial \theta} \frac{\partial \theta}{\partial \mu}+\frac{\partial \operatorname{Loss}}{\partial \mu} \\
=\frac{\partial \operatorname{Loss}}{\partial \theta}+\frac{\partial \operatorname{Loss}}{\partial \mu} \\
\frac{\partial}{\partial \rho} \operatorname{Loss}=\frac{\partial \operatorname{Loss}}{\partial \theta} \frac{\partial \theta}{\partial \rho}+\frac{\partial \operatorname{Loss}}{\partial \rho} \\
=\frac{\partial \operatorname{Loss}}{\partial \theta} \frac{\varepsilon}{1+\exp (-\rho)}+\frac{\partial \operatorname{Loss}}{\partial \rho}
\end{gathered}
$$

Please note that the standard deviations of the $\frac{\partial L o s s}{\partial \theta}$ term of the mean and the gradient are shared, and it happens to be the gradient found by the backpropagation algorithm on the normal LSTM network. Therefore, to learn the mean and standard deviation, we can calculate the gradient by backpropagation and then scale and translate it. We summarize the optimization process as seven steps in Table 2. 
Table 2. The optimization process for the Bayesian LSTM networks.

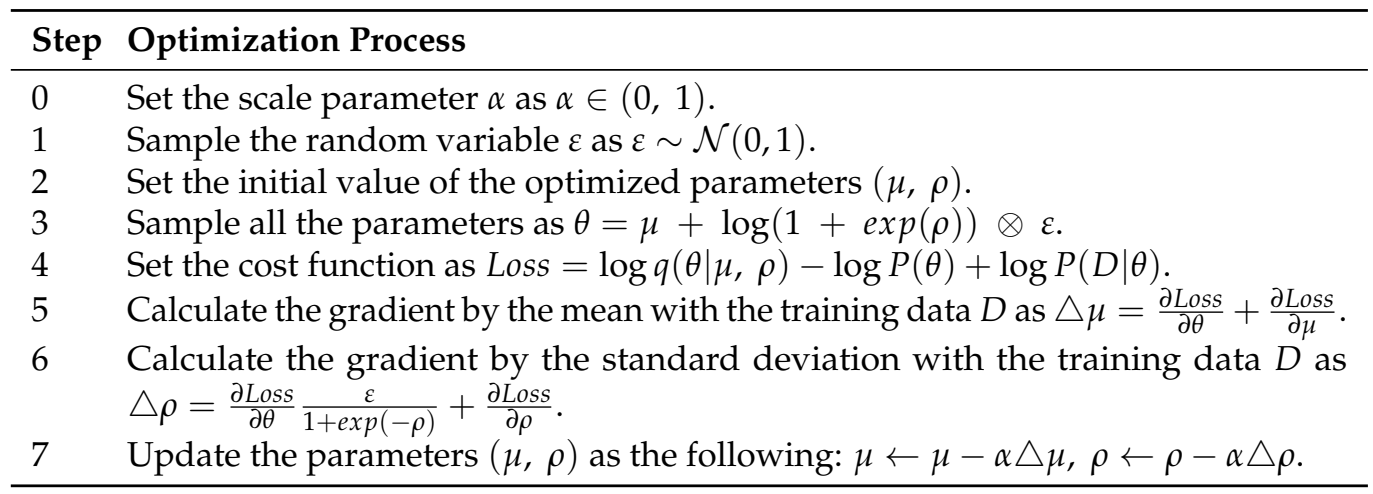

\subsection{Model Framework}

We propose a distributed prediction model combining SCC and a deep learning network for the prediction problem. The proposed model framework is shown in Figure 5, and the model consists of three main components: selection nodes, sub-predictors, and fusion nodes.

The selection node calculates the series causality of the data source and selects the variables related to the target data as the network input. For each selected input variable, a Bayesian LSTM sub-predictor is designed. Finally, we use the fusion node to fuse the prediction results of multiple sub-predictors. An artificial neural network MLP is used in the fusion node. MLP is a fully linked combination of artificially designed neurons, which applies a nonlinear activation function to model the relationship between the input and output.

\section{Experiments}

\subsection{Dataset}

Our experiments used the meteorological dataset in Shunyi District, Beijing, from 2017 to 2019. The data were measured hourly at meteorological station. The future temperature was chosen to be predicted to test the proposed model. The data set contained 1095 days for a total of 26,280 data samples to ensure sufficient training data. We selected the first $90 \%$ of the data for training and the remaining $10 \%$ for testing.

\subsection{Experimental Setup}

A PC with an Intel CORE CPU i5-4200U $1.60 \mathrm{GHz}$ and 6 GB of memory was used for the experiments. In the experiments, the default parameters in Keras and Pytorch were used for deep neural network initialization. We used the ReLU as the activation function of the Bayesian LSTM layer and the linear activation function of the MLP layer.

We set up one Bayesian LSTM layer and one MLP layer, and each layer's size was set to 24. The Adam algorithm was used for the supervised training, and the model was trained by mini-batch sampling. The model hyperparameters, such as learning and batch size, were obtained from experiments and are presented in Table 3.

Table 3. Hyperparameters for the experiments.

\begin{tabular}{lll}
\hline Layers & Design Details & Experiment Setup \\
\hline \multirow{3}{*}{ Bayesian LSTM } & Number of layers: 1 & Batch size: 30 \\
& Number of neurons: 24 & Epochs: 100 \\
& Sampling number: 4 & Learning rate: 0.001 \\
\hline \multirow{2}{*}{ MLP } & Number of layers: 1 & \\
& Number of neurons: 24 & \\
\hline
\end{tabular}




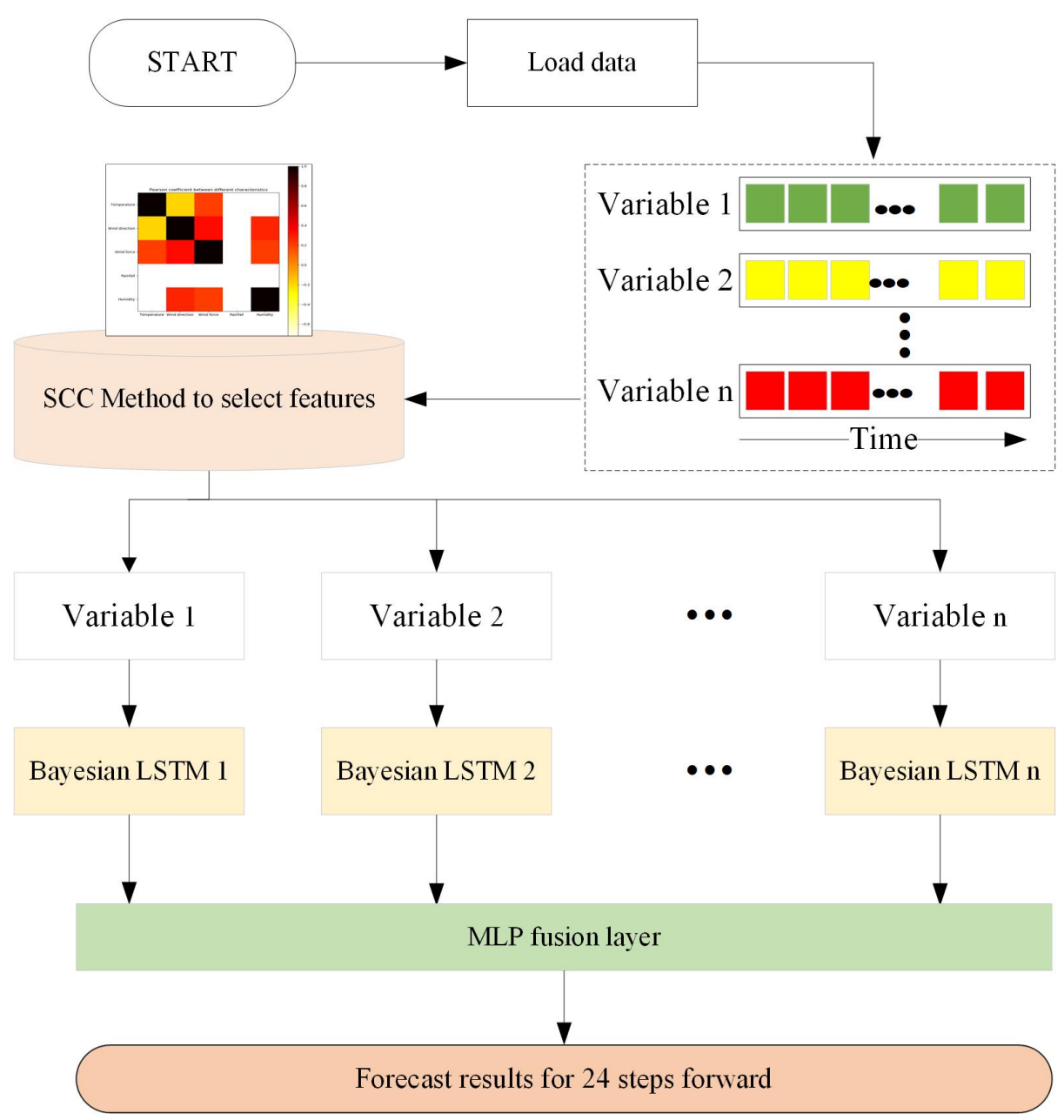

Figure 5. Model framework.

The model's performance was evaluated by the following four factors. The root-meansquared error (RMSE):

$$
R M S E=\sqrt{\frac{1}{n} \sum_{i=1}^{n}\left(y_{i}-\widehat{y}_{i}\right)^{2}}
$$

where $\widehat{y}_{i}$ is the prediction, $y_{i}$ is the ground truth, and $n$ is the number of data.

The mean-squared error (MSE) can reflect the value of the loss function of network convergence and is defined as:

$$
M S E=\frac{1}{n} \sum_{i=1}^{n}\left(y_{i}-\widehat{y}_{i}\right)^{2}
$$

The mean absolute error (MAE) and Pearson correlation coefficient (R) between the prediction and reference were also explored in the experiments.

$$
\begin{gathered}
M A E=\frac{1}{n} \sum_{i=1}^{n}\left|y_{i}-\widehat{y}_{i}\right| \\
R=\frac{\sum_{i=1}^{n}\left(y_{i}-\bar{y}_{i}\right)\left(\widehat{y}_{i}-\overline{\widehat{y}}_{i}\right)}{\sqrt{\sum_{i=1}^{n}\left(y_{i}-\bar{y}_{i}\right)^{2} \sum_{i=1}^{n}\left(\widehat{y}_{i}-\bar{y}_{i}\right)^{2}}}
\end{gathered}
$$




\subsection{Case 1}

In this case, the Bayesian LSTM model's performance is verified and causality evaluated by predicting the further temperature. We used the SCC to compare the correlation between time series variables and selected the temperature and humidity as the distributed deep model's input data. We set the time step to 24 and got a total of 24 prediction steps. The blue and red lines present the ground truth of temperature and the model's predictive results, respectively. The RMSE of the prediction is 3.203.

Figure 6 shows the comparison of the measurement data (the ground truth) and the 24 step forward prediction results. There is a light red band above and below the red line, which is the variance of the Bayesian network's result. It can be seen that the predictive trend is close to the ground truth, and most of the forecast values are within the confidence interval.

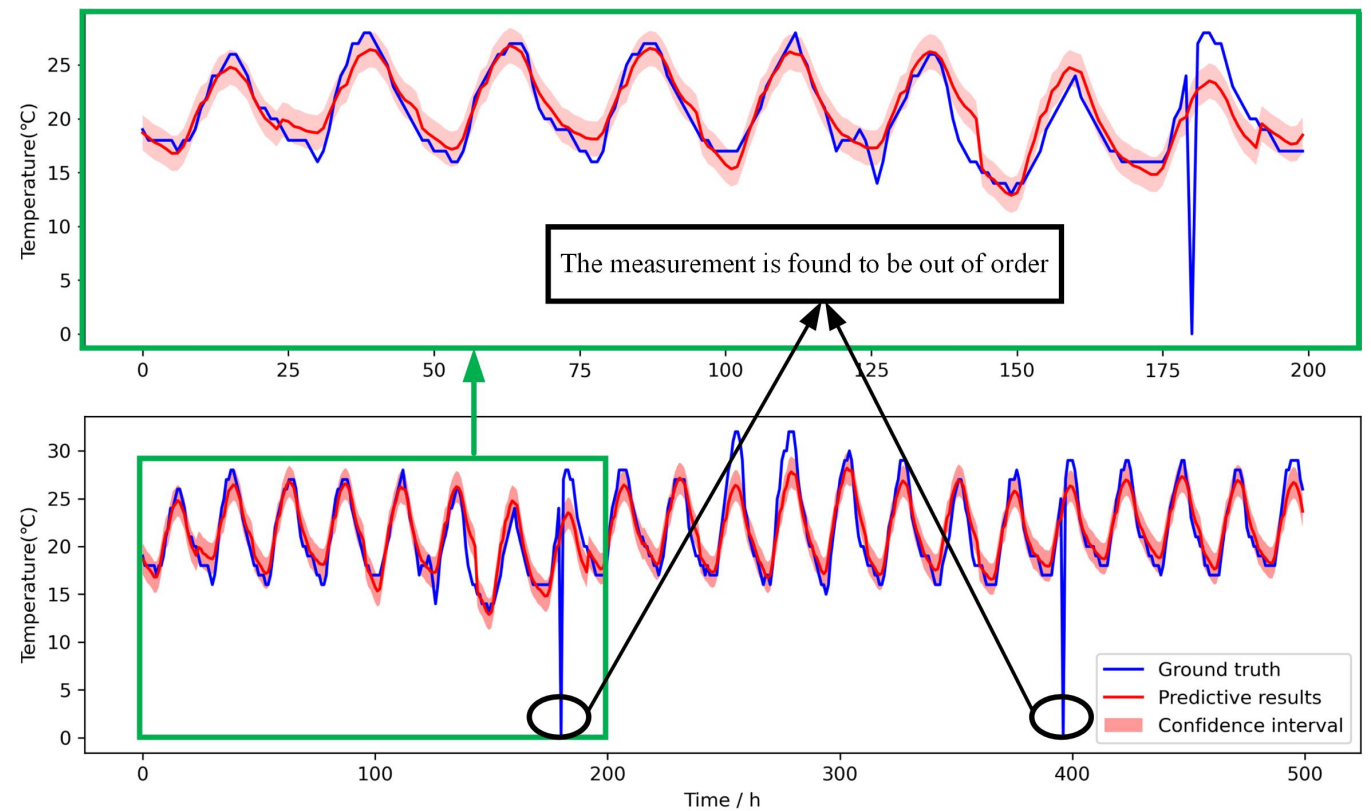

Figure 6. The prediction results of the temperature. The above picture is the prediction for the first 200 hours, which is a part of the bottom picture, in which we draw the results for about 21 days. We can see that in the bottom picture, the sensor is out of order with two hours, in which the sensor measurement data are zero. However, the prediction result effectively overcomes the sensor's failure and gives a daily temperature trend consistent with historical data.

From the actual measurement data, the prediction model's input data caused by sensor failure give the wrong measurement value. We can see that in the bottom picture, the sensor is out of order in two hours, in which the sensor measurement data are zero. However, the prediction result effectively overcomes the sensor's failure and gives a daily temperature trend consistent with historical data. However, the prediction result still maintains the correct trend, which effectively overcomes the sensor failure.

\subsection{Case 2}

In this case, we calculated the causality of the four meteorological factors in the data set and selected the best data for the network model. Because the SCC is zero between temperature and rainfall, we did not consider the rainfall data in the prediction.

The data set used to predict the temperature is four meteorological elements, i.e., historical temperature, humidity, wind force, and wind direction. We first considered two variables as the input of the network. We found that the predicted performance was different in different combinations. This performance was related to the SCC parameter. In another case, we increased the input signal to three or four. The results show that as the 
sensor input data increased, the prediction performance would not improve, but would decrease instead.

Table 4 and Figure 7 show the comparison results with two inputs. It can be seen from Table 4 that when historical temperature and humidity are set as the input, the best prediction performance can be obtained, in which the RMSE, MSE, and MAE are 3.203, 10.260 , and 2, respectively. Compared with other combinations of input, such as historical temperature and wind force and historical temperature and wind direction, the RMSE, MSE, and MAE decreased.

The larger the SCC, the more it shows that the data have more causality with respect to the target data. As shown in Table 1, the historical temperature data and humidity have the greatest correlation with the future temperature data. Therefore, using these two types of data, compared with historical temperature data as the input, we can significantly improve the prediction performance.

Table 4. Prediction performance with two inputs.

\begin{tabular}{lllll}
\hline Input Data & RMSE & MSE & MAE & R \\
\hline Historical temperature and humidity & 3.203 & 10.260 & 2.000 & 0.940 \\
Historical temperature and wind force & 3.244 & 10.525 & 2.108 & 0.937 \\
Historical temperature and wind direction & 3.400 & 11.559 & 2.250 & 0.932 \\
\hline
\end{tabular}

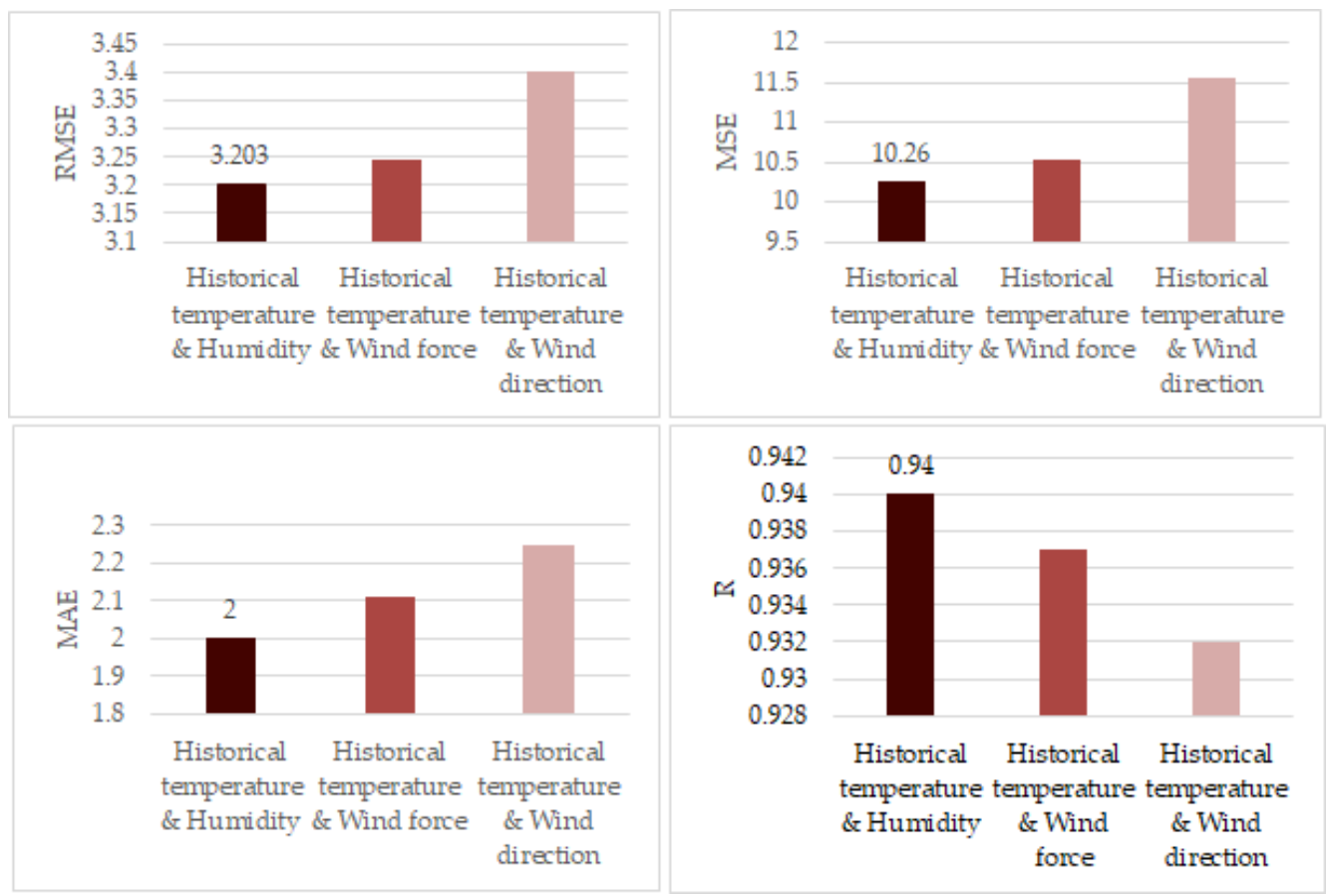

Figure 7. Comparison of prediction performance with two inputs. The input variables are historical temperature and humidity, historical temperature and wind force, and historical temperature and wind direction, respectively. We can find that when the inputs are the historical temperature and humidity, the least RMSE, MSE, and MAE and the largest R can be obtained.

Then, we increased the input variables one-by-one, adding humidity, wind force, and wind direction, separately. The performance of different numbers of inputs are shown in Table 5 and Figure 8. We can see that when there was only historical temperature as the input data, the RMSE, MSE, and MAE were 3.508, 12.305, and 2.331, respectively. Then, when two inputs were used, that is together historical temperature with humidity, the minimum prediction RMSE was 3.203. In addition, the MAE, MSE, and R were the best also. However, when the input data increased and three input data were used, the RMSE increased to 3.235. When four input data were used, the RMSE further increased to 3.230 . 
Therefore, we can conclude that the experiments show that more input data do not result in better prediction performance.

Table 5. Prediction performance with multiple inputs.

\begin{tabular}{lllll}
\hline Input Data & RMSE & MSE & MAE & R \\
\hline Historical temperature & 3.508 & 12.305 & 2.331 & 0.930 \\
Historical temperature and humidity & 3.203 & 10.260 & 2.000 & 0.940 \\
Historical temperature, humidity, and wind force & 3.235 & 10.465 & 2.014 & 0.938 \\
Historical temperature, humidity, wind force, and & 3.230 & 10.430 & 2.032 & 0.938 \\
wind direction & & & & \\
\hline
\end{tabular}

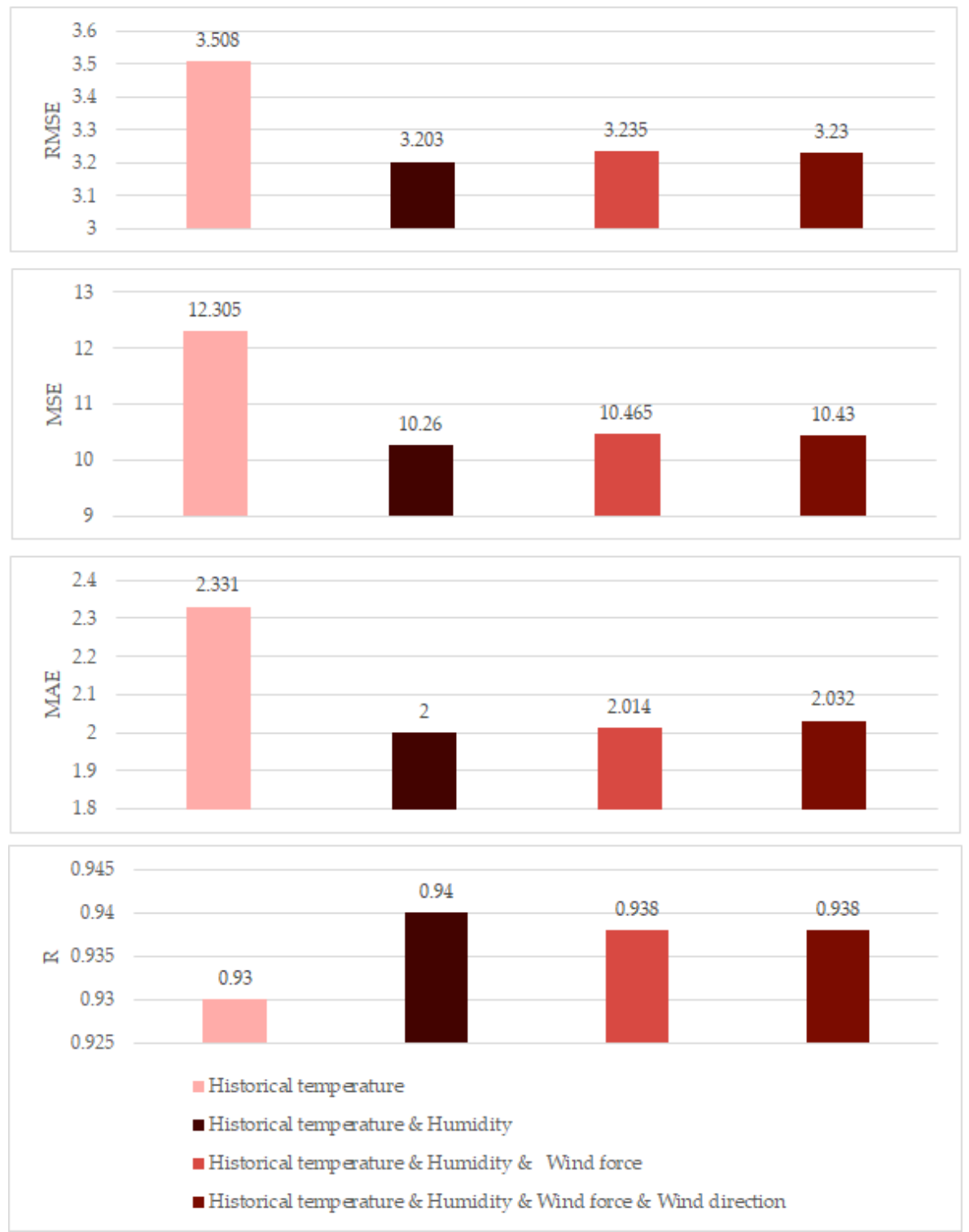

Figure 8. Comparison of prediction performance with multiple inputs. We can see that when two input variables are used, compared with one input variable, the RMSE, MSE, and MAE decrease and $\mathrm{R}$ increases, which shows that the performance is getting better. However, as the number of input variables increases, the performance becomes worse. For example, when the input variables are historical temperature, humidity, and wind force, the prediction performance worsens. Further, when we use the four input variables, the performance is the worst. 


\subsection{Case 3}

In this case, we compared other deep network models with the methods proposed in this paper. Among them, no baseline models included a feature selection process and used all features as the network input. As shown in Table 6 and Figure 9, the RMSEs of LSTM [27], GRU [28], CNN-LSTM [11], conv-LSTM [30], and the proposed Bayesian LSTM were $3.714,3.429,3.630,3.594$, and 3.203 and the MSEs were 13.797, 11.759, 13.174, 12.915, and 10.260, respectively. The MAEs were $2.467,2.137,2.406,2.344$, and 2.000, respectively. Compared with LSTM and GRU, the RMSE of the proposed Bayesian LSTM decreased by $13.76 \%$ and $6.59 \%$, and the MSE decreased by $25.64 \%$ and $12.75 \%$, while the MAE decreased by $18.93 \%$ and $6.41 \%$, respectively. Compared with other hybrid models, such as CNN-LSTM and conv-LSTM, the results show that the Bayesian LSTM was the best, obtaining the minimum RMSE of 3.203 and the least MAE of 2.000. Therefore, the Bayesian LSTM can better fit the data and had the best prediction performance.

Table 6. Prediction performance with different models.

\begin{tabular}{ccccc}
\hline Model & RMSE & MSE & MAE & R \\
\hline LSTM [27] & 3.714 & 13.797 & 2.467 & 0.927 \\
GRU [28] & 3.429 & 11.759 & 2.137 & 0.930 \\
CNN-LSTM [11] & 3.630 & 13.174 & 2.406 & 0.922 \\
Conv-LSTM [30] & 3.594 & 12.915 & 2.344 & 0.931 \\
The proposed Bayesian LSTM & 3.203 & 10.260 & 2.000 & 0.940 \\
\hline
\end{tabular}

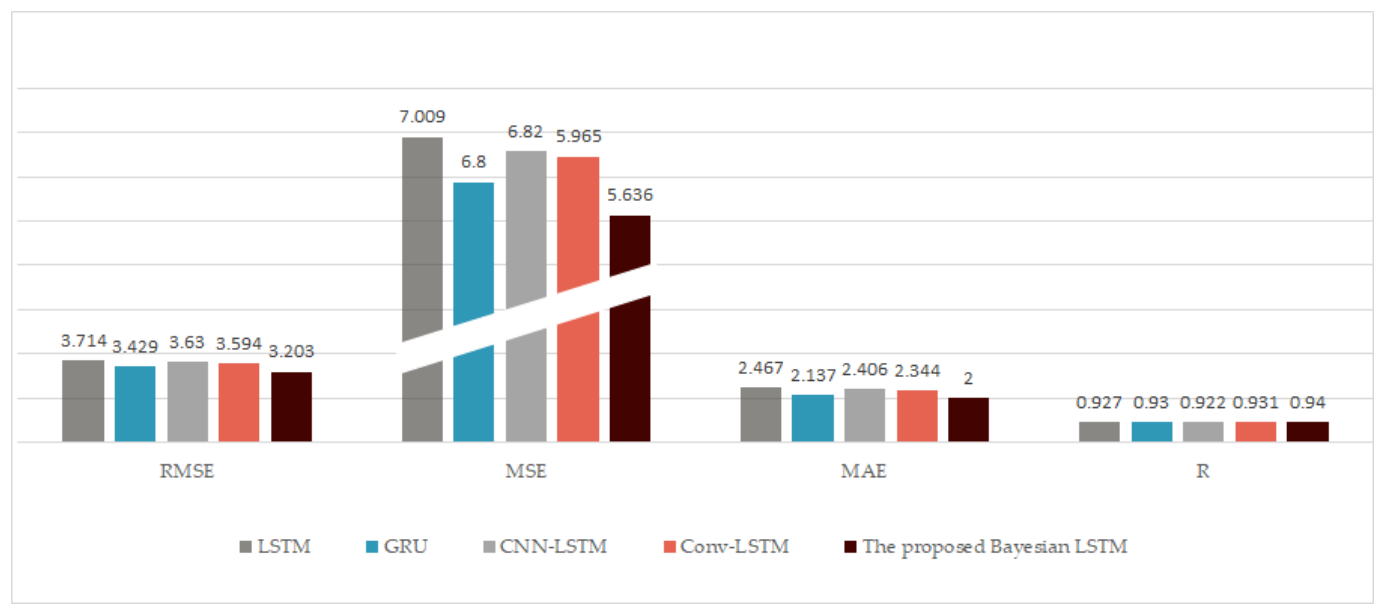

Figure 9. Comparison of the prediction performance with different sub-predictors. We can find that the proposed model with the Bayesian LSTM is the best, obtaining the least RMSE, of 2.374, MSE, and MAE and the largest $R$.

\section{Conclusions}

This article focuses on multivariate noisy measurement data modeling and prediction and proposes a distributed deep Bayesian LSTM prediction network based on causality entropy. The performance of the model was verified on real weather data sets.

In a multi-sensor system, the actual data set is usually non-linear and noisy. Therefore, analyzing the correlation between measurement from a multi-sensor system is very important for predicting. We developed the SCC to analyze the original multidimensional variables and then selected the most causal variable for the target variable. The SCC can reduce the total amount of data entered into the network, thereby reducing the computational burden of the network. It also reduces errors caused by unnecessary input. 
As we all know, neural networks have a strong ability to fit nonlinearity. However, we found that the measurement data from the multi-sensor system have complex noise. We used the Bayesian LSTM to reduce the influence of noise on the neural network. The model was modeled by weight sampling, and then, the average was taken to obtain a more stable output.

In future research, we can consider other causality analysis methods. We will also replace the MLP with other fusion methods to reduce the network model's parameters for the fusion results. The proposed approaches in the paper can combine other parameter estimation algorithms [32,64-67] to study the parameter identification problems of linear and nonlinear systems with different disturbances [68-72], and to build the soft sensor models and prediction models and can be applied to other fields [73-77] such as signal processing and process control systems.

Author Contributions: Conceptualization, X.-B.J.; data curation, Y.-T.B. and J.-L.K.; formal analysis, T.-L.S. and Y.-T.B.; methodology, X.-H.Y.; software, X.-H.Y.; supervision, L.W.; validation, T.-L.S.; visualization, D.-N.Y.; writing, original draft, X.-H.Y.; writing, review and editing, X.-B.J. All authors have read and agreed to the published version of the manuscript.

Funding: This work was supported in part by the National Key Research and Development Program of China No. 2020YFC1606801, the National Natural Science Foundation of China Nos. 61903009 and 61903008, the Beijing Municipal Education Commission Nos. KM201910011010 and KM201810011005, the Young Teacher Research Foundation Project of BTBU No. QNJJ2020-26, the Defense Industrial Technology Development Program No. 6142006190201, and the Beijing excellent talent training support project for young top-notch team No. 2018000026833TD01.

Data Availability Statement: The data presented in this study are available on request from the corresponding author.

Conflicts of Interest: The authors declare no conflict of interest.

\section{References}

1. Rubio, F.; Valero, F.; Llopis-Albert, C. A review of mobile robots: Concepts, methods, theoretical framework, and applications. Int. J. Adv. Robot. Syst. 2019, 16, 1-22. [CrossRef]

2. Zhao, Z.; Wang, X.; Yao, P.; Bai, Y. A health performance evaluation method of multirotors under wind turbulence. Nonlinear Dyn. 2020, 102. [CrossRef]

3. Zhang, X; Zhao, Z.; Wang, Z; Wang, X. Fault detection and identification method for quadcopter based on airframe vibration signals. Sensors 2021, 21, 581. [CrossRef]

4. Jin, X.; Yu, X.; Wang, X. Deep Learning Predictor for Sustainable Precision Agriculture Based on Internet of Things System. Sustainability 2020, 12, 1433. [CrossRef]

5. Jin, X.; Yang, N.; Wang, X. Hybrid Deep Learning Predictor for Smart Agriculture Sensing Based on Empirical Mode Decomposition and Gated Recurrent Unit Group Model. Sensors 2020, 20, 1334. [CrossRef]

6. Senthilkumar, R.; Venkatakrishnan, P.; Balaji, N. Intelligent based novel embedded system based IoT enabled air pollution monitoring system. Microprocess. Microsyst. 2020, 77, 103172. [CrossRef]

7. Jin, X.; Yang, N.; Wang, X. Integrated Predictor Based on Decomposition Mechanism for PM2.5 Long-Term Prediction. Appl. Sci. 2019, 9, 4533. [CrossRef]

8. Jin, X.; Sun, S.; Wei, H.; Yang, F. Advances in Multi-Sensor Information Fusion: Theory and Applications 2017. Sensors 2018, 18, 1162. [CrossRef] [PubMed]

9. Lee, D.; Kim, K. Recurrent neural network-based hourly prediction of photovoltaic power output using meteorological information. Energies 2019, 12, 215. [CrossRef]

10. Jin, X.; Yang, N.; Wang, X. Deep hybrid model based on EMD with classification by frequency characteristics for long-term air quality prediction. Mathematics 2020, 8, 214. [CrossRef]

11. Huang, C.J.; Kuo, P.H. A deep CNN-LSTM model for particulate matter (PM2.5) forecasting in smart cities. Sensors 2018, 18, 2220. [CrossRef] [PubMed]

12. Bai, Y.; Wang, X.; Sun, Q. Spatio-temporal prediction for the monitoring-blind area of industrial atmosphere based on the fusion network. Int. J. Environ. Res. Public Health 2019, 16, 3788. [CrossRef] [PubMed]

13. Bai, Y.; Jin, X.; Wang, X.; Wang, X.; Xu, J. Dynamic correlation analysis method of air pollutants in spatio-temporal analysis. Int. J. Environ. Res. Public Health 2020, 17, 360. [CrossRef] [PubMed]

14. Ziemann, T.; Peri, H.; Singh, A. System and method for enhancing trust for person-related data sources. U.S. Patent 10,542,043, 21 January 2020. 
15. Runge, J.; Nowack, P.; Kretschmer, M.; Flaxman, S.; Sejdinovic, D. Detecting and quantifying causal associations in large nonlinear time series datasets. Sci. Adv. 2019, 5, eaau4996. [CrossRef]

16. Bai, Y.; Jin, X.; Wang, X.; Su, T.; Kong, J.; Lu, Y. Compound autoregressive network for prediction of multivariate time series. Complexity 2019, 2019, 9107167. [CrossRef]

17. Rendon-Sanchez, J.F.; de Menezes, L.M. Structural combination of seasonal exponential smoothing forecasts applied to load forecasting. Eur. J. Oper. Res. 2019, 275, 916-924. [CrossRef]

18. Gautam, S. A novel moving average forecasting approach using fuzzy time series data set. J. Control. Autom. Electr. Syst. 2019, 30, 532-544. [CrossRef]

19. Messner, J.W.; Pinson, P. Online adaptive lasso estimation in vector autoregressive models for high dimensional wind power forecasting. Int. J. Forecast. 2019, 35, 1485-1498. [CrossRef]

20. Alsharif, M.; Younes, M.; Kim, J. Time series ARIMA model for prediction of daily and monthly average global solar radiation: The case study of Seoul, South Korea. Symmetry 2019, 11, 240. [CrossRef]

21. Zhang, X.; Wang, J.; Gao, Y. A hybrid short-term electricity price forecasting framework: Cuckoo search-based feature selection with singular spectrum analysis and SVM. Energy Econ. 2019, 81, 899-913. [CrossRef]

22. Chitsazan, M.A.; Fadali, M.S.; Trzynadlowski, A.M. Wind speed and wind direction forecasting using echo state network with nonlinear functions. Renew. Energy 2019, 131, 879-889. [CrossRef]

23. Ren, Y.; Mao, J.; Liu, Y. A novel dbn model for time series forecasting. IAENG Int. J. Comput. Sci. 2017, 44, 79-86.

24. Sulaiman, J.; Wahab, S.H. Heavy rainfall forecasting model using artificial neural network for flood prone area. In IT Convergence and Security 2017; Springer: Singapore, 2018; pp. 68-76.

25. Izonin, I.; Tkachenko, R.; Verhun, V.; Zub, K. An approach towards missing data management using improved GRNN-SGTM ensemble method-ScienceDirect. Eng. Sci. Technol. Int. J. 2020, in press.

26. Min, K.; Kim, D.; Park, J.; Huh, K. RNN-based path prediction of obstacle vehicles with deep ensemble. IEEE Trans. Veh. Technol. 2019, 10, 10252-10256. [CrossRef]

27. Sundermeyer, M.; Schlüter, R.; Ney, H. LSTM neural networks for language modeling. In Proceedings of the Thirteenth Annual Conference of the International Speech Communication Association, Portland, OR, USA, 9-13 September 2012; pp. $194-197$.

28. Wang, Y.; Liao, W.; Chang, Y. Gated recurrent unit network-based short-term photovoltaic forecasting. Energies 2018, 11, 2163. [CrossRef]

29. Tang, X.; Dai, Y.; Wang, T. Short-term power load forecasting based on multi-layer bidirectional recurrent neural network. IET Gener. Transm. Distrib. 2019, 13, 3847-3854. [CrossRef]

30. Qi, Y.; Li, Q.; Karimian, H.; Liu, D. A hybrid model for spatiotemporal forecasting of PM2.5 based on graph convolutional neural network and long short-term memory. Sci. Total. Environ. 2019, 664, 1-10. [CrossRef]

31. Tian, C.; Ma, J.; Zhang, C. A deep neural network model for short-term load forecast based on long short-term memory network and convolutional neural network. Energies 2018, 11, 3493. [CrossRef]

32. Xu, L.; Ding, F. Iterative parameter estimation for signal models based on measured data. Circuits Syst. Signal Process. 2018, 37, 3046-3069. [CrossRef]

33. Xu, L. The parameter estimation algorithms based on the dynamical response measurement data. Adv. Mech. Eng. 2017, 9, 1687814017730003. [CrossRef]

34. Ding, F.; Wang, X.H.; Mao, L.; Xu, L. Joint state and multi-innovation parameter estimation for time-delay linear systems and its convergence based on the Kalman filtering. Dig. Signal Proc. 2017, 62, 211-223. [CrossRef]

35. Ding, F.; Xu, L.; Zhu, Q. Performance analysis of the generalised projection identification for time-varying systems. IET Control. Theory Appl. 2016, 10, 2506-2514. [CrossRef]

36. Ding, J.; Cao, Z.X.; Chen, J.Z.; Jiang, G.P. Weighted parameter estimation for Hammerstein nonlinear ARX systems. Circuits Syst. Signal Proc. 2020, 39, 2178-2192. [CrossRef]

37. Ding, F.; Xu, L.; Meng, D.; Jin, X.B.; Alsaedi, A.; Hayat, T. Gradient estimation algorithms for the parameter identification of bilinear systems using the auxiliary model. J. Comput. Appl. Math. 2020, 369, 112575. [CrossRef]

38. Pan, J.; Jiang, X.; Wan, X.K.; Ding, W. A filtering based multi-innovation extended stochastic gradient algorithm for multivariable control systems. Int. J. Control Autom. Syst. 2017, 15, 1189-1197. [CrossRef]

39. Zhang, X. Recursive parameter estimation and its convergence for bilinear systems. IET Control. Theory Appl. 2020, 14, 677-688. [CrossRef]

40. Li, M.H.; Liu, X.M. The least squares based iterative algorithms for parameter estimation of a bilinear system with autoregressive noise using the data filtering technique. Signal Process. 2018, 147, 23-34. [CrossRef]

41. Zhang, X.; Liu, Q.Y. Recursive identification of bilinear time-delay systems through the redundant rule. J. Frankl. Inst. 2020, 357, 726-747. [CrossRef]

42. $\mathrm{Xu}, \mathrm{L}$. Parameter estimation algorithms for dynamical response signals based on the multi-innovation theory and the hierarchical principle. IET Signal Process. 2017, 11, 228-237. [CrossRef]

43. Waldmann, P. On the use of the pearson correlation coefficient for model evaluation in genome-wide prediction. Front. Genet. 2019, 10, 899. [CrossRef] [PubMed]

44. Amarkhil, Q.; Elwakil, E.; Hubbard, B. A meta-analysis of critical causes of project delay using spearman's rank and relative importance index integrated approach. Can. J. Civ. Eng. 2020, Just-IN. [CrossRef] 
45. Duan, S.; Yang, W.; Wang, X. Grain pile temperature forecasting from weather factors: A support vector regression approach. In Proceedings of the 2019 IEEE/CIC International Conference on Communications in China (ICCC), Changchun, China, 11-13 August 2019; pp. 255-260. [CrossRef]

46. Ciulla, G.; D'Amico, A. Building energy performance forecasting: A multiple linear regression approach. Appl. Energy 2019, 253, 113500. [CrossRef]

47. Jing, B.; Qian, Z.; Pei, Y.; Wang, J. Ultra short-term PV power forecasting based on ELM segmentation model. J. Eng. 2017, 2017, 2564-2568. [CrossRef]

48. Lin, C.Y.; Chang, Y.S.; Chiao, H.T. Design a Hybrid Framework for Air Pollution Forecasting. In Proceedings of the 2019 IEEE International Conference on Systems, Man and Cybernetics (SMC), Bari, Italy, 6-9 October 2019; pp. 2472-2477.

49. Gao, D.; Zhou, Y.; Wang, T.; Wang, Y. A Method for predicting the remaining useful life of lithium-ion batteries based on particle filter using Kendall rank correlation coefficient. Energies 2020, 13, 4183. [CrossRef]

50. Contreras-Reyes, J.E.; Hernández-Santoro, C. Assessing granger-causality in the southern humboldt current ecosystem using cross-spectral methods. Entropy 2020, 22, 1071. [CrossRef]

51. Podobnik, B.; Stanley, H.E. Detrended cross-correlation analysis: a new method for analyzing two nonstationary time series. Phys. Rev. Lett. 2008, 100, 084102. [CrossRef] [PubMed]

52. Jin, X.; Zhang, J.; Su, T.; Bai, Y.; Kong, J.; Wang, X. Wavelet-deep optimized model for nonlinear multi-component data forecasting. Comput. Intell. Neurosci. 2021, accept.

53. Jin, X.; Wang, H.; Wang, X. Deep-learning prediction model with serial two-level decomposition based on bayesian optimization. Complexity 2020, 2020, 4346803 [CrossRef]

54. Wang, H.; Yeung, D.Y. A survey on Bayesian deep learning. ACM Comput. Surv. (CSUR) 2020, 53, 1-37. [CrossRef]

55. Mukhopadhyay, P.; Mallick, S. Bayesian deep learning for seismic facies classification and its uncertainty estimation. Soc. Explor. Geophys. 2019, 2488-2492. [CrossRef]

56. Zhang, R.; Li, C.; Zhang, J. Cyclical stochastic gradient MCMC for Bayesian deep learning. arXiv 2019, arXiv:1902.03932.

57. Jin, X.; Lian, X.; Su, T.; Shi, Y.; Miao, B. Closed-loop estimation for randomly sampled measurements in target tracking system. Math. Probl. Eng. 2014, 2014, 315908.

58. Jin, X.; Du, J.; Bao, J. Target tracking of a linear time invariant system under irregular sampling. Int. J. Adv. Robot. Syst. 2012, 9, 219-230.

59. Li, G.; Yang, L.; Lee, C.G.; Wang, X.; Rong, M. A Bayesian deep learning RUL framework integrating epistemic and aleatoric uncertainties. IEEE Trans. Ind. Electron. 2020, 1. [CrossRef]

60. Harper, R.; Southern, J. A Bayesian deep learning framework for end-to-end prediction of emotion from heartbeat. IEEE Trans. Affect. Comput. 2020. [CrossRef]

61. Abdi, H. The Kendall rank correlation coefficient. In Encyclopedia of Measurement and Statistics, Salkind, N.J., Ed; SAGE Publications Inc.: Thousand Oaks, CA, USA, 2007; pp. 508-510.

62. Lopez Quintero, F.O.; Contreras-Reyes, J.E.; Wiff, R.; Arellano-Valle, R.B. Flexible Bayesian analysis of the von bertalanffy growth function with the use of a log-skew-t distribution. Fish. Bull. 2017, 115 13-26. [CrossRef]

63. Bouhlel, N.; Dziri, A. Kullback-Leibler divergence between multivariate generalized gaussian distributions. IEEE Signal Process. Lett. 2019, 26, 1021-1025. [CrossRef]

64. Xu, L.; Xiong, W.L.; Alsaedi, A.; Hayat, T. Hierarchical parameter estimation for the frequency response based on the dynamical window data. Int. J. Control Autom. Syst. 2018, 16, 1756-1764. [CrossRef]

65. Gu, Y.; Liu, J.; Li, X.; Chou, Y.; Ji, Y. State space model identification of multirate processes with time-delay using the expectation maximization. J. Frankl. Inst. 2019, 356, 1623-1639. [CrossRef]

66. Xu, L.; Ding, F.; Zhu, Q.M. Hierarchical Newton and least squares iterative estimation algorithm for dynamic systems by transfer functions based on the impulse responses. Int. J. Syst. Sci. 2019, 50, 141-151. [CrossRef]

67. Xu, L.; Ding, F.; Lu, X.; Wan, L.J.; Sheng, J. Hierarchical multi-innovation generalised extended stochastic gradient methods for multivariable equation-error autoregressive moving average systems. IET Control. Theory Appl. 2020, 14, 1276-1286. [CrossRef]

68. Pan, J.; Ma, H.; Zhang, X.; Liu, Q.; Ding, F.; Chang, Y.; Sheng, J. Recursive coupled projection algorithms for multivariable output-error-like systems with coloured noises. IET Signal Process. 2020, 14, 455-466. [CrossRef]

69. Xu, L.; Ding, F.; Wan, L.J.; Sheng, J. Separable multi-innovation stochastic gradient estimation algorithm for the nonlinear dynamic responses of systems. Int. J. Adapt. Control Signal Process. 2020, 34, 937-954. [CrossRef]

70. Zhang, X.; Ding, F.; Alsaadi, F.E.; Hayat, T. Recursive parameter identification of the dynamical models for bilinear state space systems. Nonlinear Dyn. 2017, 89, 2415-2429. [CrossRef]

71. Zhang, X.; Xu, L.; Ding, F.; Hayat, T. Combined state and parameter estimation for a bilinear state space system with moving average noise. J. Frankl. Inst. 2018, 355, 3079-3103. [CrossRef]

72. Gu, Y.; Zhu, Q.; Nouri, H. Bias compensation-based parameter and state estimation for a class of time-delay nonlinear state-space models. IET Control. Theory Appl. 2020, 14, 2176-2185. [CrossRef]

73. Zhang, X.; Ding, F.; Xu, L.; Yang, E. State filtering-based least squares parameter estimation for bilinear systems using the hierarchical identification principle. IET Control. Theory Appl. 2018, 12, 1704-1713. [CrossRef]

74. Wang, L.J.; Ji, Y.; Wan, L.J.; Bu, N. Hierarchical recursive generalized extended least squares estimation algorithms for a class of nonlinear stochastic systems with colored noise. J. Frankl. Inst. 2019, 356, 10102-10122. [CrossRef] 
75. Zhang, X.; Ding, F.; Xu, L.; Yang, E.F. Highly computationally efficient state filter based on the delta operator. Int. J. Adapt. Control Signal Process. 2019, 33, 875-889. [CrossRef]

76. Fan, Y.M.; Liu, X.M. Two-stage auxiliary model gradient-based iterative algorithm for the input nonlinear controlled autoregressive system with variable-gain nonlinearity. Int. J. Robust Nonlinear Control 2020, 30, 5492-5509. [CrossRef]

77. Zhang, X.; Ding, F.; Yang, E.F. State estimation for bilinear systems through minimizing the covariance matrix of the state estimation errors. Int. J. Adapt. Control Signal Process. 2019, 33, 1157-1173. [CrossRef] 